Volume 19, No 3 International Journal of Radiation Research, July 2021

\title{
Dosimetric evaluation of intensity modulated radiation therapy for different duty cycles of the gated beam delivery
}

\author{
K. Kaviarasu ${ }^{1,2}$, N. Arunai Nambi Raj ${ }^{*}$, K. Krishna Murthy ${ }^{2}$ \\ ${ }^{1}$ Department of Physics, School of Advanced Sciences, VIT, Vellore-632014, India \\ ${ }^{2}$ Department of Radiation Oncology, Krishna Institute of Medical Sciences, Secunderabad-500003, India \\ ${ }^{3}$ Centre for Biomaterials, Cellular and Molecular Theranostics (CBCMT), VIT, Vellore - 632014, India
}

\section{- Original article}

\section{*Corresponding authors: \\ N. Arunai Nambi Raj, Ph.D., E-mail:}

narunainambiraj@vit.ac.in

Revised: June 2020

Accepted: July 2020

Int. J. Radiat. Res., July 2021; 19(3): 669-683

DOI: $10.29252 /$ ijrr.19.2.669

\begin{abstract}
Background: Aim of this study was to evaluate the extent of the error that the gating system incorporates into an intensity-modulated radiation therapy (IMRT) delivery for the different duty cycles of beam gated treatments (beaminterruption) by comparing the gamma between the dose planes. Materials and Methods: Respiratory motion patterns was recorded in the real-time position management (RPM) software, which controls the triggering of the linear accelerator for the beam ON/OFF based on the predefined gating window. 10 IMRT plans consisting of 60 IMRT fields were delivered for three different duty cycles $(20 \% \mathrm{DC}, 30 \% \mathrm{DC}$ and $40 \% \mathrm{DC})$ of gated and non-gated delivery. Planar dose measurements of IMRT delivery were performed with the commercially available two-dimensional ion chamber array and portal dosimetry. Gamma evaluation was carried out for the three different duty cycles of gated delivery with that of the reference of non-gated delivery, and the measured dose planes of gated and non-gated delivery were gamma analyzed with the treatment planning system (TPS) dose planes. Multileaf collimator (MLC) dynalog files were acquired and analyzed for the different duty cycles of gated and non-gated IMRT deliveries. Results: Gamma between the gated and non-gated dose planes were found within the $3 \%$ deviation. Area gamma for the gated and non-gated delivery to the reference of TPS dose planes were found within the deviation of $6 \%$. Conclusion: Gamma comparison of the gated delivery with the reference of non-gated delivery results demonstrated that increasing the duty cycle reduced the deviation between the gated and non-gated delivery.
\end{abstract}

Keywords: Duty cycle, gating techniques, gamma evaluation, intensity-modulated radiation therapy, respiratory motion management.

\section{INTRODUCTION}

The goal of radiotherapy is to minimize the absorbed dose to normal tissue while maximizing the dose to the target volume. The advent of intensity-modulated radiation therapy (IMRT) and volumetric-modulated arc therapy (VMAT) has allowed for dose escalation to the target volume and reduction in dose to the surrounding normal tissue. These techniques have been successfully applied for the radiotherapy treatment of tumors in various sites. Precise radiotherapy techniques such as IMRT and VMAT require more accurate patient positioning and knowledge of the tumor position during irradiation. Respiration-induced tumor motion may be a major source of error in the delivered dose distribution in the regions of the thorax and abdomen for these types of precise radiotherapy techniques ${ }^{(1-3)}$. To overcome this conventionally, the tumor motion must be taken into account by adding an internal margin around the clinical target volume (CTV), in order to create the internal target volume (ITV). 
Positioning uncertainties are then added to create the planning target volume (PTV) for the planning $(4,5)$. However, this strategy has limitations. For tumors with significant respiratory motion, this can lead to the irradiation of very large volumes of normal tissues to a high dose that could increase the risk of unacceptable complications, and therefore limit the possibility of dose escalation. If the respiratory motion is managed by gating techniques, the conformal dose distribution generated by IMRT and VMAT can be realized in the abdominal/thoracic region tumors (6-10). Several strategies are currently used to reduce the effects of respiratory motion in the thorax and abdominal regions, which include breath hold techniques, forced shallow breathing with abdominal compression, integration of respiratory movements into the treatment planning (4DCT scan), automatic breath control techniques, respiratory gating techniques, and real-time tumor tracking techniques. These strategies are grouped under the general term of respiratory-gated radiation therapy. Regardless of which of the several strategies is used in respiratory-gated radiotherapy treatments, benefits are expected in terms of geometric precision and dosimetric improvements (11-12). In respiratory-gated treatments, internal organ movements are correlated with external surrogates of the respiratory motion. Therefore, in most cases, some type of external surrogate can be selected to properly gate the radiation delivery.

Of the above mentioned strategies, respiratory gating techniques involve the delivery of radiation (during both imaging and treatment delivery) within a particular portion (phase or amplitude) of the patient's breathing cycle, commonly the term referred as a "gate." The ratio of the time spent by the signal within the gate to the overall treatment time is referred to as the duty cycle (13).These techniques restrict the range of positions of the tumor and internal normal tissue structures during the imaging and radiation delivery. This virtual restriction of the tumor position gives us a reduced internal margin component of the PTV and potentially reduces the normal tissue toxicity, thereby allowing dose escalation and hence increased tumor control.

Dynamic treatment delivery log files (DynaLogs) are accessible on Varian linear accelerators (Varian Medical Systems, Palo Alto, USA), and include details of the cumulative dose fraction, beam on status, segment number, as well as the expected and actual MLC leaf positions for the dynamic IMRT delivery. The delivery accuracy of IMRT can be quantified by the MLC position errors. The actual MLC positions and delivered fraction of the monitor units (MU) of IMRT delivery were extracted from the DynaLog files (14-15). These extracted data can be used to verify the accuracy of MLC leaf positions, which enables determining the accuracy of IMRT delivery. Several studies have independently validated the accuracy of the DynaLog file beam data using different quality assurance (QA) methods, such as film, diode array, and portal imaging (16-18). In gated IMRT, the movement of DMLC leaves is frequently interrupted according to the duty cycle of the treatment. Our aim was to determine whether these frequent beam interruptions (gated delivery) affect the planned fluence or not. To analyze that, we acquired and analyzed the DynaLog files for gated and non-gated deliveries in this study.

The quality assurance of linear accelerators generally does not include the evaluation of gated IMRT delivery. Extensive quality assurances are needed to evaluate the performance of gated IMRT delivery. Several authors have investigated the compatibility of IMRT with gated delivery. Moreover, several studies have investigated the effects of small MU segments on beam delivery, MU-dose linearity for small MUs, beam flatness, MLC positioning accuracy, and gating window size for gated radiation therapy treatment delivery (19-24).

The aim of this study was to evaluate the extent of the error that the gating system incorporates into an intensity-modulated radiation therapy (IMRT) delivery for the different duty cycles of beam-gated treatments (beam-interruption) by comparing the gamma between the dose planes. In this study, all our gated delivery measurements were 
compared with the non-gated delivery, and this comparison provides an evaluation of the extent of the error the gating system incorporates into an IMRT delivery for different duty cycles of gated treatments.

\section{MATERIALS AND METHODS}

\section{Linear accelerator and gating system}

The delivery of respiratory-gated dynamic IMRT was tested on a Novalis Tx (Varian Medical Systems, Palo Alto, USA) linear accelerator equipped with a high-definition multileaf collimator (HD 120 MLC) in this study. The linear accelerator was interfaced with a real-time position management (RPM) respiratory gating system (Varian Medical Systems, Pala Alto, USA) for the gated treatments. The linear accelerator is capable of delivering dual-energy photon beams of 6 and $15 \mathrm{MV}$ X-ray beams with dose rate ranging from 100 to $600 \mathrm{MU} / \mathrm{min}$. The IMRT beams analyzed in this study were planned for $6 \mathrm{MV}$ photon beams with a dose rate of $400 \mathrm{MU} / \mathrm{min}$ (clinically used dose rate in our center) in the Eclipse TPS (Varian Medical Systems, Palo Alto, USA). The DMLC IMRT mode, which is often used in clinics, was used in this study.

The RPM system consists of an infrared video camera, an infrared illuminator ring, a reflective marker block (six-dot or two-dot), and a workstation with the system control software. The RPM system tracks the respiratory cycles of the patient through a reflective marker block placed on a surface of the patient's body (usually the external surface of the body with the greatest breathing motion). The reflective marker block is used as an external surrogate of the respiratory motion. The reflective marker block reflects the infrared light from the infrared illuminator ring of a camera to the charge-coupled device (CCD) of the same camera. The camera is connected to a workstation linked to the accelerator. The movement of the reflectors induced by breathing is analyzed in real time by the RPM software, which controls the triggering of the linear accelerator for beam ON/OFF state based on a predefined gating window. The beam is then interrupted between each breath and the total dose is delivered in small fractions of a few monitor units.

In this study, we used a RPM motion phantom supplied by Varian medical systems to generate breathing patterns. The motion phantom has an elliptical wheel that rotates at approximately 10 rotations per minute and creates a sinusoidal movement of the markers with a displacement of $2.0 \mathrm{~cm}$. This motion corresponds to a breathing cycle of $6.0 \mathrm{~s}$. For all the gated delivery measurements, the beam is gated with three different duty cycles (DC) of $20 \%, 30 \%$, and $40 \%$. The approximate beam-on times of $1.2,1.8$, and $2.4 \mathrm{~s}$ and approximate monitor units of 8,12, and $16 \mathrm{MU}$ for a $400 \mathrm{MU} / \mathrm{min}$ dose rate were used for the $20 \%, 30 \%$, and $40 \%$ duty cycles, respectively. We placed the phantom as per the measurement setup shown in figure 1. The RPM system tracks the motion of the marker and records the breathing curve. The gating system sends the signal to the linear accelerator to trigger the beam hold-off, when the marker moves beyond the preset gating duty cycle.

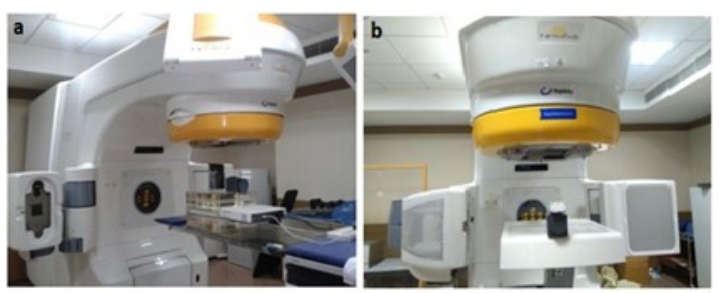

Figure 1. Measurement setup in linear accelerator for gated delivery using RPM phantom with (a) IMatriXX evaluation ion chamber 2D array (b) EPID.

\section{Quality assurance of gated delivery}

To validate that the gated delivery (beam interruption) does not alter the beam characteristics and dose delivery accuracy, several measurements were carried out using phantoms with and without gating. In this study, all dosimetric measurements were performed under the following two scenarios: 1 . stationary phantom, no beam gating (non-gated delivery); 2. Stationary phantom and beam gating (gated delivery). An amorphous silicon electronic portal imaging device (aS1000 EPID), IMatriXX evaluation ion chamber $2 \mathrm{D}$ array with a multicube phantom (iba-Dosimetry, $\mathrm{GmbH}$, 
Germany), SP34 solid water phantom (iba-Dosimetry, GmbH, Germany), FC65G Farmer ionization chamber (iba-Dosimetry, $\mathrm{GmbH}$, Germany), cc13 compact ionization chamber (iba-Dosimetry, GmbH, Germany), and Dose 1 electrometer (iba-Dosimetry, GmbH, Germany) were used in this study for the profile and point dose measurements. MLC transmission, DLG measurements, beam profile analysis, DMLC output, and MLC position accuracy for the gated delivery (20\% DC, 30\% DC and 40\% DC) are measured and the results are compared with those of non-gated delivery to validate the gated delivery in this study.

\section{a. MLC transmission}

MLC transmission is an important characteristic of the MLC modeling in a TPS, and is defined as the transmission of radiation through the MLC. MLC transmission generally has two components, which are the radiation that is transmitted through and attenuated by the full thickness of the leaf, and the radiation that is transmitted through the space between adjacent leaves. MLC transmission measurements were performed with a $0.65 \mathrm{cc}$ Farmer chamber in an SP34 solid water phantom of size $30 \times 30 \times 20 \mathrm{~cm}^{3}$. The source-tophantom distance was $100 \mathrm{~cm}$ and the depth of measurement was $5 \mathrm{~cm}$. The leaf edges were offset be $7 \mathrm{~cm}$ from the central axis to avoid the leakage contribution due to rounded leaf edge. MLC transmission measurements were performed for the gated delivery $(20 \% \mathrm{DC}, 30 \%$ DC and 40\% DC) and non -gated delivery in this study.

\section{b. Dosimetric leaf gap measurements}

A Novalis Tx linear accelerator employs an MLC with rounded leaf ends; this design characteristic allows rectilinear leaf motion while maintaining a consistent penumbra width, irrespective of the leaf position. Consequently, there is a discrepancy between the geometric field width (light field) and the dosimetric field width owing to leakage through the rounded leaf ends, which is defined as the dosimetric leaf gap (DLG). This value is used in dose calculations to correctly model field modulation.
In this study, we measured the DLG for the gated and non-gated delivery by the sweeping gap method (25-27). The Varian MLC shaper software (Varian Medical Systems, Palo Alto, USA) package was used to create such DMLC sweeping gap fields (28). The sweeping gap field moves from -60 to $+60 \mathrm{~mm}$ at a constant speed with respect to MU to deliver the dose. DLG measurements were performed with FC65G Farmer ionization chamber in an SP34 solid water phantom of size $30 \times 30 \times 20 \mathrm{~cm}^{3}$. The source-to-phantom distance was $100 \mathrm{~cm}$ and the depth of the measurement was $5 \mathrm{~cm}$. DLG measurements were performed for the gated delivery $(20 \% \mathrm{DC}, 30 \% \mathrm{DC}$ and $40 \%$ DC) and non -gated delivery in this study.

\section{c. Beam profiles - open field gamma analysis}

Open field beam profiles for different field sizes of gated and non-gated deliveries were measured using an IMatriXX ion chamber 2D array (iba-Dosimetry, GmbH, Germany) with a multicube phantom. The measurement setup had a source-to-detector distance (SDD) of $100 \mathrm{~cm}$. The IMatriXX consists of 1020 air-vented ionization chambers with a sensitive volume of $0.08 \mathrm{~cm}^{3}$ at a water-equivalent depth of $3 \mathrm{~mm}$ (effective point of measurement) arranged in a $32 \times 32$ grid (excluding the four corner positions) over an active area of $24.4 \times 24.4 \mathrm{~cm}^{2}$. The spatial resolution, given by the center-to-center distance of two neighboring detectors, is 7.62 $\mathrm{mm}$. The different field sizes of gated beam profiles at the isocenter planes were acquired and gamma was verified for $1 \%$ dose $/ 1 \mathrm{~mm}$ distance with that of non-gated beam profiles using the OmniPro IMRT software (iba-Dosimetry, GmbH, Germany) package in this study.

\section{d. DMLC output}

DMLC output data were collected using a 0.65 -cc Farmer ionization chamber (FC65G) and a Dose 1 electrometer for the $10 \times 10 \mathrm{~cm}^{2}$ field size and the DMLC sweeps of 2, 4, 6, 10,14, 16, and 20 $\mathrm{mm}$. The Varian MLC shaper software package was used to create such DMLC sweeping gap fields. Output measurements were performed in an SP34 solid water phantom of dimensions $30 \times 30 \times 20 \mathrm{~cm}^{3}$ with a source-to-surface distance

Int. J. Radiat. Res., Vol. 19 No. 3, July 2021 
of $100 \mathrm{~cm}$. The ionization chamber was kept at a depth of $5 \mathrm{~cm}$ and irradiated with 6-MV X-rays for 200MU with the Novalis $\mathrm{Tx}$ linear accelerator. The raw meter readings for the gated delivery were compared to those for the non-gated delivery. Because of the relative characteristics of the measurements, uncorrected meter readings from the electrometer were used to determine the ratio between the gated and non-gated deliveries.

\section{e. Static MLC (SMLC) positioning accuracy}

To examine the effect of gating on the static MLC positioning accuracy, a picket fence MLC test pattern was delivered with and without gating on an amorphous silicon (aS1000) portal imager (Varian Medical Systems, Palo Alto, USA) . The picket fence test MLC patterns were created with the MLC shaper software (Varian Medical Systems, Palo Alto, USA) package; the test pattern consists consecutive leaf movements of 2 -mm-wide strips of length $18 \mathrm{~cm}$ spaced at $1.5-\mathrm{cm}$ intervals. The acquired portal images were gamma-analyzed for the $1 \%$ dose/ $1 \mathrm{~mm}$ distance in a portal dosimetry workspace of the ARIA integrating system, keeping the non-gated picket fence as a reference. In addition to that, to verify the MLC positions, we acquired the crossline profiles at the isocenter and $5 \mathrm{~cm}$ above and below the isocenter of the acquired portal images of three different duty cycles of gated (20\% DC, 30\% DC and 40\% DC) and non-gated deliveries.

\section{f. Dynamic MLC (DMLC) positioning accuracy}

To examine the effect of gating on the dynamic MLC positioning accuracy, different MLC sweep gaps were delivered with and without gating on an amorphous silicon (aS1000) portal imager. The DMLC sweeps were created by the MLC shaper software package. DMLC sweep fields of $2,4,6,10,14,16$, and 20 $\mathrm{mm}$ were irradiated to the EPID to measure the planar fluence at the isocenter, while maintaining the measurement setup with a SDD of $100 \mathrm{~cm}$. Portal images at the isocenter of non-gated delivery were gamma-analyzed with the gated delivery for the criteria of $1 \%$ depth dose (DD) / $1 \mathrm{~mm}$ distance to agreement (DTA) using the portal dosimetry software of the ARIA (Varian Medical Systems, Palo Alto, USA) integrated system. The fractions of the points in the measurement area with gamma $\leq 1.0$ for $1 \%$ DD to $1 \mathrm{~mm}$ DTA were calculated. DMLC position accuracy measurements were performed for the gated delivery (20\%DC, 30\%DC and 40\% DC) and non -gated delivery.

\section{g. QA of gated IMRT plan delivery}

To verify whether the IMRT gated delivery alters the beam characteristic and dose delivery accuracy, the following measurements were carried out in phantom with and without gating. We measured and compared the dose planes using an IMatriXX evaluation ion chamber 2D array and EPID.

In this study, 10 IMRT plans consisting of 60 IMRT fields were taken. In the Eclipse Treatment Planning System (TPS), two verification plans were created for each IMRT plans.

1. The verification plans of the IMRT fields were created on an IMatriXX evaluation 2D Array with a locally fabricated multicube phantom. The dose planes at the isocentre for each field are calculated in the Eclipse TPS. The calculated dose planes at isocentre for each field in the TPS were transferred into the OmniPro IMRT software package. The verification plan was irradiated on an IMatriXX evaluation 2D array with a locally fabricated multicube phantom and the dose planes at the isocentre were acquired using the OmniPro IMRT software for three different duty cycles $(20 \%, 30 \%$, and $40 \%$ DC) of gated delivery and non-gated delivery for twice.

2. The portal dosimetry verification plans were created in the Eclipse TPS. The portal dosimetry quality assurance plans were irradiated on an aS1000 EPID, which was mounted on the Novalis Tx linear accelerator. The portal dose images were acquired for each IMRT field for three different duty cycles $(20 \%$, $30 \%$, and $40 \%$ DC) of gated delivery and non-gated delivery twice.

We chose the gamma analysis method to analyze the accuracy of the gated and non-gated deliveries, and we found the fractions of the points in the measurement area with gamma $\leq$ 
1.0. Gamma, a quantitative dose evaluation tool, is the magnitude of the minimum vector from a reference dose distribution to a measurement distribution. Gamma is scaled by the selection of criteria on the dose and distance, such that if gamma is scaled by greater than one, the measurement fails with respect to the distribution $(29,30)$. The gamma between different duty cycles of gated and non-gated IMRT delivery to the reference of the TPS-calculated dose planes for the criteria of $3 \%$ dose to $3 \mathrm{~mm}$ distance were calculated for both the verification methods to verify the dosimetric accuracy of the IMRT delivery over the planned delivery in the TPS. The non-gated delivery represents the reference case to which the other three gated deliveries are compared for the criteria of $1 \%$ dose to $1 \mathrm{~mm}$ distance to quantify the degree of the error incorporated by the gating (beam interruption) to the IMRT delivery.

\section{h. IMRT plan point dose verification}

An SP34 solid water phantom (iba-Dosimetry, GmbH, Germany) was used to measure the point dose at the isocentre with an FC65G Farmer type ion chamber connected to a Dose 1 electrometer (iba-Dosimetry, GmbH, Germany). The dimensions of the SP34 phantom are $300 \mathrm{~mm} \times 300 \mathrm{~mm} \times 200 \mathrm{~mm}$ (consisting of 20 SP34 water slabs of thickness $10 \mathrm{~mm}$ ) and it is composed of the material RW3. The phantom plan was created in the Eclipse TPS by superimposing the patient IMRT fields into a phantom, and the gantry, collimator, and couch angles were set to $0^{\circ}$. Phantom plans were calculated for an SDD of $100 \mathrm{~cm}$ and a depth of 5 $\mathrm{cm}$ for the calculation grid of $2.5 \mathrm{~mm}$ using the anisotropic analytical algorithm (AAA). Point dose measurements were carried out for three different duty cycles of gated delivery and non-gated delivery in the NovalisTx linear accelerator using the abovementioned setup geometry. The ratios of the point dose (mean dose over the contoured volume) of the gated IMRT delivery to that of the non-gated IMRT delivery were calculated. All the 60 IMRT fields, point dose at isocentre were measured for the gated (20\% DC, 30\% DC and 40\% DC) and non-gated delivery.

\section{i. DynaLog file analysis}

A DynaLog file is a record of the actual dose fraction (dose dynamic) or gantry angle (arc dynamic) versus the actual MLC leaf positions from either a dynamic treatment or a segmental treatment, generated in ASCII format. A dynamic treatment is a treatment during which the MLC leaves, collimator, or gantry move while the beam is on and both the speed of the leaves and the dose rate are continually adjusted by the MLC control system. The DynaLog data are acquired every $50 \mathrm{~ms}$ by the MLC controller. DynaLog file viewer (DFV) is a software package (Varian Medical Systems, Palo Alto, USA) that allows us to view data from DynaLog files in graphical formats. DFV analysis of the DynaLog file gives the results in two formats: 1 . Error histogram; 2. Error root mean square (RMS). The error histogram shows information of all the leaf position deviations. DFV creates a histogram with a tally of the number of leaf position deviations within each bin. The data are divided into three bins: less than $0.5 \mathrm{~mm}, 0.5 \mathrm{~mm}$ to $<1.00 \mathrm{~mm}$, and $1.00 \mathrm{~mm}$ and above. The error histogram bin boundaries represent position deviations of the MLC at the isocentre plane. Error RMS shows the calculated RMS value for the leaf deviations. DFV calculates the RMS values for the leaf position deviations of individual leaves using the equation 1 :

LeafErrorRMS $=\sqrt{\frac{\sum_{t=1}^{n}(\text { LeafPlanPost } t \text { LeafActualPos })^{2}}{n}}$

Here; $t$ is the data sample index and $n$ is the total number of samples.

The DynaLog file data contain all the leaf positions during treatment. However, for the error histogram and RMS calculations, the DFV software only considers the leaf if it or the opposing leaf moves during the treatment. The error RMS data contain the details of the average leaf error RMS and maximum leaf error RMS values for the each MLC bank (MLC bank A side and MLC bank B side) (15).

In this study, we acquired and analyzed the DynaLog files for the different MLC Sweep fields $(2,4,6,10,16$ and $20 \mathrm{~mm}$ sweeps) for the gated (20\% DC, 30\% DC and 40\% DC) and non-gated deliveries in this study to find the MLC position

Int. J. Radiat. Res., Vol. 19 No. 3, July 2021 
errors. DynaLog files of the 60 IMRT fields $(10$ plans) were acquired for the gated $(20 \%$ DC, $30 \%$ DC and 40\% DC) and non-gated deliveries in this study were analyzed to the error histogram (MLC positional errors) and the leaf error RMS.

\section{Data entry and statistical analysis}

Quality assurance of gated IMRT plan delivery measurements were done twice for the 60 fields of gated (20\% DC, 30\% DC and 40\% DC) and non-gated delivery for both the QA verification methods (ImatriXX Evaluation and Portal dosimetry) and the data were entered in the excel sheet and found average of the measurements for both the QA methods individually. We used paired t-test based on SPSS 25.0 software to compare the QA results of IMRT plan delivery of each gated beam delivery over the non-gated beam delivery, and the p-value of $<0.05$ were taken as statistically significant.

DynaLog files were collected for 60 IMRT fields twice for gated (20\% DC, 30\% DC and $40 \%$ DC) and non-gated delivery. The DynaLogs were analyzed in the DFV software for the leaf error histogram and leaf RMS values. Data were compared using paired t-test for the gated deliveries to the reference of non-gated delivery. The analysis was done using the SPSS software version 25.0. A p-value of $<.05$ was taken as statistically significant.

\section{RESULTS}

\section{QA of Gated delivery}

\section{a. MLC transmission and Dosimetric leaf gap measurements}

MLC transmission and DLG measurements were carried for three different duty cycles of gated and non-gated delivery and the results are shown in table 1 . The MLC transmissions measured with and without gating showed less than $0.3 \%$ difference between them. The DLG values showed less than $0.7 \%$ difference between the gated and non-gated deliveries. We found that the MLC transmission and DLG values were almost equal for the three different duty

Int. J. Radiat. Res., Vol. 19 No. 3, July 2021 cycles of gated and non-gated deliveries.

Table 1. MLC transmission and dosimetric leaf gap values for the $6 \mathrm{MV} \mathrm{X}$-ray beam of the Novalis Tx linear accelerator for gated and non-gated deliveries.

\begin{tabular}{|c|c|c|}
\hline $\begin{array}{c}\text { Gated Duty } \\
\text { Cycle (\%) }\end{array}$ & $\begin{array}{c}\text { MLC Transmission } \\
\text { (in \%) }\end{array}$ & $\begin{array}{c}\text { Dynamic Leaf Gap } \\
\text { (DLG) (in } \mathbf{~ m m} \text { ) }\end{array}$ \\
\hline Non-gated & 1.120 & 0.697 \\
\hline 20 & 1.123 & 0.700 \\
\hline 30 & 1.120 & 0.702 \\
\hline 40 & 1.121 & 0.698 \\
\hline
\end{tabular}

\section{b. Beam profiles - open field gamma analysis}

Beam profiles for different field sizes of gated and non-gated deliveries were measured with an IMatriXX ion chamber 2D array with a multicube phantom. The measured beam profiles were gamma-analyzed in the OmniPro IMRT software for the criteria of $1 \%$ dose / $1 \mathrm{~mm}$ distance, using the non-gated measurement as a reference. The gamma (area gamma $\leq 1.0$ ) values are tabulated in table 2 . From the obtained data, we found that good agreement was observed between the gated and non-gated deliveries. The open field beam profile gamma analysis results ensure that the flatness and symmetry are closely matching with the gated and non-gated beam deliveries. For the gamma values with the criteria of $1 \%$ dose difference to $1 \mathrm{~mm}$ distance, more than $99 \%$ percent of the gamma measurements points pass between the gated and non-gated deliveries for the different open field sizes measured in this study. This pass percentage ensures that the gated delivery beam flatness and symmetry closely agree with the non-gated delivery and that the gated delivery does not alter the beam flatness and symmetry.

Table 2. Gamma values (area gamma $\leq 1.0$ ) of different duty cycles of gated delivery over non-gated delivery for different field sizes.

\begin{tabular}{|c|c|c|c|}
\hline \multirow{2}{*}{ Field Size (in $\mathbf{~ c m}^{2}$ ) } & \multicolumn{3}{|c|}{ Area Gamma $\leq 1.0$} \\
\cline { 2 - 4 } & $20 \%$ DC & $30 \%$ DC & $40 \%$ DC \\
\hline $4 \times 4$ & 99.65 & 99.64 & 99.70 \\
\hline $6 \times 6$ & 99.63 & 99.72 & 99.77 \\
\hline $8 \times 8$ & 99.21 & 99.59 & 99.79 \\
\hline $10 \times 10$ & 99.08 & 99.62 & 99.44 \\
\hline $12 \times 12$ & 99.05 & 99.18 & 99.62 \\
\hline $15 \times 15$ & 99.22 & 99.52 & 99.73 \\
\hline $18 \times 18$ & 99.18 & 99.25 & 99.69 \\
\hline
\end{tabular}




\section{c. DMLC output}

Gated and non-gated central axis DMLC output data were collected using a 0.65-cc farmer ionization chamber and a Dose 1 electrometer for a field size of $10 \times 10 \mathrm{~cm}^{2}$ for the different DMLC sweeps of 2, 4, 6, 10, 14, 16 and $20 \mathrm{~mm}$. All the results have been normalized to the non-gated measurements for their respective sweeps. The ratios of the DMLC output values for the three different duty cycles to the non-gated delivery are shown in table 3 . The mean and SD of the ratio between the gated and non-gated deliveries are $1.0047 \pm 0.001$. This result indicates that the output deviation between the gated and non-gated deliveries is less than $0.5 \%$. This deviation is unremarkable, and it shows that the gated delivery does not notably change the output of the linear accelerator.

Table 3. DMLC output ratio for different MLC sweeps between the gated and non-gated deliveries.

\begin{tabular}{|c|c|c|c|}
\hline \multirow{2}{*}{ Sweep fields } & \multicolumn{3}{|c|}{$\begin{array}{r}\text { DML output ratio between the } \\
\text { gated \& non-gated delivery }\end{array}$} \\
\cline { 2 - 4 } & $\mathbf{2 0 \% ~ D C}$ & $\mathbf{3 0 \%}$ DC & $\mathbf{4 0 \%}$ DC \\
\hline $2 \mathrm{~mm}$ sweep & 1.0062 & 1.0055 & 1.0037 \\
\hline $4 \mathrm{~mm}$ sweep & 1.0057 & 1.0055 & 1.0052 \\
\hline $6 \mathrm{~mm}$ sweep & 1.0062 & 1.0058 & 1.0058 \\
\hline $10 \mathrm{~mm}$ sweep & 1.0052 & 1.0052 & 1.0047 \\
\hline $14 \mathrm{~mm}$ sweep & 1.0042 & 1.0033 & 1.0027 \\
\hline $16 \mathrm{~mm}$ sweep & 1.0036 & 1.0029 & 1.0026 \\
\hline $20 \mathrm{~mm}$ sweep & 1.0032 & 1.0031 & 1.0026 \\
\hline
\end{tabular}

\section{d. SMLC positioning accuracy}

To verify that the MLC positioning accuracy was not affected adversely by the gating operation, a picket fence MLC test pattern were delivered with and without gating on an amorphous silicon (aS1000) portal imager. Using the portal dosimetry workspace of the ARIA integrating system (Varian Medical Systems, Palo Alto, USA), the portal images were gamma-analyzed for the criteria of $1 \%$ dose to $1 \mathrm{~mm}$ distance, keeping the non-gated picket fence as a reference. The resulting gamma values (area gamma $\leq 1.0$ ) are 99.5\%, 99.4\%, and $99.6 \%$ for $20 \%$ DC, $30 \%$ DC, and $40 \%$ DC, respectively. Figure 2, shows the crossline profiles (at isocentre and $5 \mathrm{~cm}$ above and below to the isocentre) of the picket fence test for the gated and gated deliveries. From the figure, it is seen that gating did not introduce any change in the width of the picket fence test, which confirms that gating does not change the MLC positioning accuracy. The results demonstrate that there were no significant changes in the MLC positional errors during the gated delivery over the non-gated delivery.

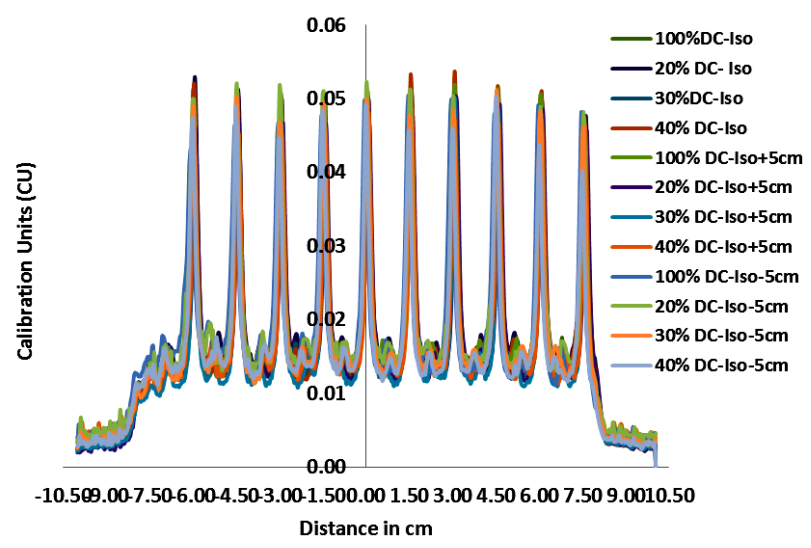

Figure 2. Static MLC position accuracy using picket fence test pattern for the gated and non-gated deliveries.

\section{e. DMLC positional accuracy}

DMLC sweep field fluence of gated and non-gated deliveries were measured with the EPID. The measured fluence were gammaanalyzed in the portal dosimetry software for the criteria of $1 \%$ dose / $1 \mathrm{~mm}$ distance, keeping the non-gated measurement as a reference. The gamma values (area gamma $\leq 1.0$ ) are tabulated in table 4 . The portal dosimetry results of the gamma analysis of gated deliveries using the non-gated delivery as a reference showed that less than $5 \%$ of the gamma analyzing points deviated for the gamma criteria of $1 \%$ dose difference to $1 \mathrm{~mm}$ distance. From the data, we observed that the $20 \%$ duty cycle showed slightly more deviation than the other two duty cycles of gated delivery for all the sweep fields analyzed in this study. We also observed a trend that the increase in duty cycle increased the pass percentage of the gamma values. The change in the sweep width results in greater gamma deviation in smaller sweep gaps compared with larger sweep gaps. 
Table 4. DMLC sweep field gamma values (area gamma $\leq 1.0$ ) of different duty cycles of gated delivery over non-gated delivery with EPID portal dosimetry.

\begin{tabular}{|c|c|c|c|}
\hline \multirow{2}{*}{} & \multicolumn{3}{|c|}{ Area Gamma $\leq \mathbf{1 . 0}$} \\
\cline { 2 - 4 } & $\mathbf{2 0 \%}$ DC & $\mathbf{3 0 \%}$ DC & 40\% DC \\
\hline $2 \mathrm{~mm}$ sweep & 95.17 & 95.45 & 96.62 \\
\hline $4 \mathrm{~mm}$ sweep & 95.79 & 96.23 & 97.97 \\
\hline $6 \mathrm{~mm}$ sweep & 96.24 & 96.94 & 97.88 \\
\hline $10 \mathrm{~mm}$ sweep & 96.61 & 97.45 & 98.52 \\
\hline $14 \mathrm{~mm}$ sweep & 96.82 & 97.81 & 98.86 \\
\hline $16 \mathrm{~mm}$ sweep & 97.21 & 98.21 & 99.15 \\
\hline $20 \mathrm{~mm}$ sweep & 97.89 & 98.55 & 99.43 \\
\hline
\end{tabular}

\section{f. QA of gated IMRT plan delivery}

Ten IMRT plans consisting of 60 IMRT fields were taken, and those 60 fields were delivered for three different duty cycles of gating and non-gating deliveries onto the IMatriXX evaluation 2D array and EPID.

Figures 3and 4 show scatter plots of percentage of the field area with a gamma value $\geq 1.0$ (area gamma $\geq 1.0$ ) values for each of the 60 IMRT fields considered in our study. These were evaluated for the criteria of $3 \%$ dose / $3 \mathrm{~mm}$ distance for the gated and non-gated deliveries using the IMatriXX evaluation 2D array with the OmniPro IMRT software and EPID dosimetry, respectively. Our results demonstrate that the gated and non-gated deliveries of IMRT delivery is quietly gamma agreeing with the planned delivery. The obtained gamma evaluation values are less than the acceptable values (area gamma $\geq 1.0$, is $7 \%$ for the $3 \% \mathrm{DD}, 3 \mathrm{~mm}$ DTA) kept at our center. From the results of the gamma evaluation, we found that the gated and non-gated delivery dose planes are in good agreement with the TPS calculated dose planes, which shows that the gating does not change the dose delivery accuracy of the IMRT. In this analysis, we measured area gamma $\geq 1.0$ instead of area gamma $\leq 1.0$, in order to plot the graphs with a smaller scale.

Figures 5 and 6 shows the scatter plot of the percentage of the field area with a gamma value $\geq 1.0$ (area gamma $\geq 1.0$ ) values for the three different duty cycles of gated delivery with respect to that of non-gated delivery. These were evaluated for the criteria of $1 \%$ dose $/ 1 \mathrm{~mm}$ distance using the IMatriXX evaluation 2D array with the OmniPro IMRT software and EPID dosimetry, respectively. We analyzed with the lesser criteria of gamma analyzing parameters of $1 \%$ dose / $1 \mathrm{~mm}$ distance, to find the extent of error incorporated by the gating system to the IMRT delivery. From the scatter plot data, we observed a trend in the gamma evaluation results of the different duty cycles of gated delivery, that the duty cycle increases, the area gamma $\geq 1.0$ decreases, which shows that the agreement between the measured and the calculated dose planes is increasing. In this analysis, for both QA measurements, we found that the gamma pass percentage is more than $97 \%$ for the three duty cycles of the gated delivery and we found that the mean deviation of the gamma pass percentage is less than $99 \%$ (area gamma $\geq 1.0$ ) for all three duty cycles of the gated delivery and the values are tabulated in table 5 . We observed the same trend of the results in this analysis, such that the gamma pass percentage increases as the duty cycle increases. This shows that the increasing duty cycle increases agreement of the gated delivery with the non-gated delivery, and the error caused by the gated delivery decreases. We have statistically analyzed the gamma evaluation results keeping the non-gated as a reference using the paired t test, we found that there is no statistical deviation for the three different duty cycles of gated delivery and the p-value calculated was $<0.001$. This statistical analysis ensures that the gamma evaluation results of gated deliveries are not significantly differ from the non-gated delivery.

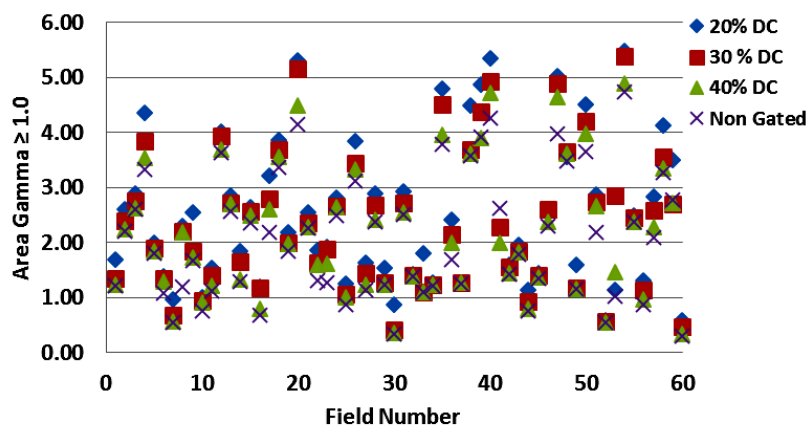

Figure 3. Gamma evaluation between the TPS-calculated dose planes and measured dose planes of IMatriXX by OmniPro IMRT analysis for all IMRT fields of gated and non-gated deliveries for the criteria of $3 \%$ dose $/ 3 \mathrm{~mm}$ distance. 


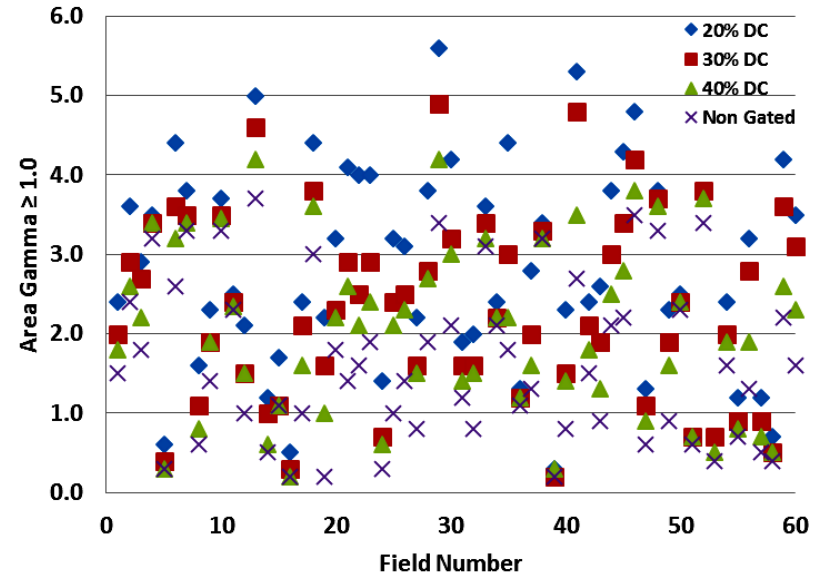

Figure 4. Gamma evaluation between the TPS-calculated portal dose and measured portal dose of EPID by portal dosimetry analysis for all IMRT fields of gated and non-gated deliveries for the criteria of $3 \%$ dose $/ 3 \mathrm{~mm}$ distance.

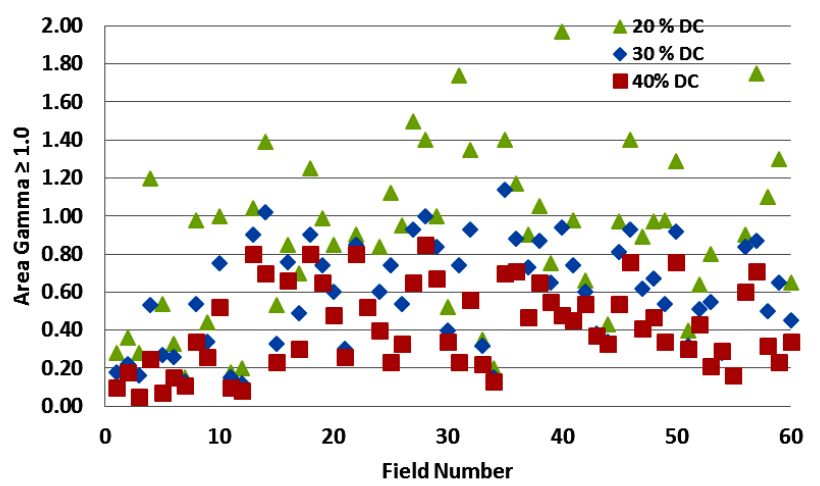

Figure 5. Gamma evaluation between the measured different duty cycles of gated dose planes versus measured non-gated dose planes of IMatriXX by OmniPro IMRT analysis for all IMRT fields for the criteria of $1 \%$ dose $/ 1 \mathrm{~mm}$ distance.

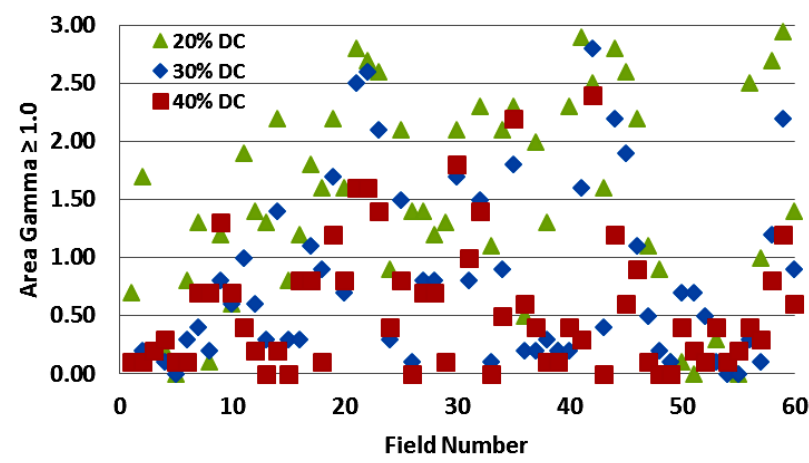

Figure 6. Gamma evaluation between the measured different duty cycles of gated portal dose planes versus measured non-gated portal dose planes of EPID by portal dosimetry analysis for all IMRT fields for the criteria of $1 \%$ dose / $1 \mathrm{~mm}$ distance.

\section{g. IMRT plan point dose verification}

Point dose measurements were carried out for three different duty cycles of gated delivery and non-gated deliveries for the 10 plans consisting of 60 IMRT fields. The doses at the isocentre were calculated and the point dose ratios between gated and non-gated IMRT delivery were determined. The results are tabulated in table 5, which shows the mean and SD of the ratio between the gated and non-gated delivery point dose measurements. It is clear from the readings that the point dose ratios between the gated and non-gated deliveries approach unity as the duty cycle increases. IMRT point dose measurement results also follow the same trend, the difference between the gated and non-gated deliveries reduces as the duty cycle increases.

Table 5. IMRT planar gamma analysis - mean and standard deviation values of area gamma $\geq 1.0$ for gated and non-gated deliveries and ratio of point dose between gated and non-gated deliveries for the 60 IMRT fields.

\section{Gamma Analysis - Area Gamma $\geq 1.0$ mean \pm standard deviation}

Gated vs TPS calculated Dose plane Gamma analysis (3\% Dose / $3 \mathrm{~mm}$ distance) - IMatriXX

\begin{tabular}{|c|c|c|c|}
\hline Non Gated & $\mathbf{2 0 \%}$ Duty Cycle & $\mathbf{3 0 \%}$ Duty Cycle & $\begin{array}{c}\mathbf{4 0 \%} \text { Duty Cy- } \\
\text { cle }\end{array}$ \\
\hline $2.06 \pm 1.13$ & $2.55 \pm 1.32$ & $2.36 \pm 1.26$ & $2.19 \pm 1.18$ \\
\hline $\begin{array}{c}\text { Paired t test } \\
\text { - p-value }\end{array}$ & $<0.001$ & $<0.001$ & $<0.001$ \\
\hline
\end{tabular}

Gated vs TPS calculated Dose plane Gamma analysis (3\% Dose / $3 \mathrm{~mm}$ distance) - EPID

\begin{tabular}{|l|l|l|l|} 
Non Gated & $20 \%$ Duty Cycle $30 \%$ Duty Cycle $40 \%$ Duty Cycle
\end{tabular}

\begin{tabular}{|c|c|c|c|}
\hline $1.66 \pm 1.02$ & $2.81 \pm 1.31$ & $2.33 \pm 1.18$ & $2.05 \pm 1.07$ \\
\hline $\begin{array}{c}\text { Paired t test } \\
\text { - p-value }\end{array}$ & $<0.001$ & $<0.001$ & $<0.001$
\end{tabular}

Gated vs Non-gated Dose plane Gamma analysis (1\% Dose / $1 \mathrm{~mm}$ distance) - IMatriXX

\begin{tabular}{|l|l|l|l|} 
Non Gated & $20 \%$ Duty Cycle & $30 \%$ Duty Cycle $40 \%$ Duty Cycle \\
\hline
\end{tabular} \begin{tabular}{l|l|l}
$0.84 \pm 0.44$ & $0.59 \pm 0.27$ & $0.42 \pm 0.22$
\end{tabular}

Gated vs Non-gated Dose plane Gamma analysis (1\% Dose / $1 \mathrm{~mm}$ distance) -EPID

\begin{tabular}{|l|l|l|l|} 
Non Gated & $20 \%$ Duty Cycle & $30 \%$ Duty Cycle $40 \%$ Duty Cycle
\end{tabular}

\begin{tabular}{|c|c|c|c|}
\hline- & $0.95 \pm 0.86$ & $0.79 \pm 0.75$ & $0.58 \pm 0.57$ \\
\hline \multicolumn{4}{|c|}{ Ratio of Point Dose } \\
\hline Non Gated & $20 \%$ Duty Cycle & $30 \%$ Duty Cycle & $40 \%$ Duty Cycle \\
\hline- & $1.0027 \pm 0.0017$ & $1.0014 \pm 0.0019$ & $1.0012 \pm 0.0015$ \\
\hline
\end{tabular}

Int. J. Radiat. Res., Vol. 19 No. 3, July 2021 


\section{h. DynaLog file analysis}

\section{h1. MLC sweep files DynaLog analysis}

DMLC sweep fields DynaLog data were collected during the measurements of the DLG, sweep field gamma analysis, and DMLC output for the different sweeps of the gated and non-gated deliveries. In total 84 DynaLog files were acquired and analyzed (three times each measurements), for the gated and non-gated deliveries for the seven sweeps $(2,4,6,10,14$, 16 and $20 \mathrm{~mm}$ ) fields. The DynaLog files were analyzed in the DFV utility software (Varian Medical Systems, Palo Alto, USA) and the mean values of the percentage of MLC position error counts of the two bins (bin 1: $>0.5 \mathrm{~mm}$ and bin 2: 0.5 to $<1.0 \mathrm{~mm}$ ) are tabulated in table 6 .
Table 6. Percentage of MLC position error counts within the bin for different sweep fields of gated and non-gated deliveries by DynaLog file analysis.

\begin{tabular}{|c|c|c|c|c|c|c|}
\hline \multirow[t]{2}{*}{$\begin{array}{l}\text { Gating Duty } \\
\text { Cycle / Sweep } \\
\text { Fields }\end{array}$} & \multicolumn{4}{|c|}{$\begin{array}{c}\text { Percentage of MLC } \\
\text { position error counts } \\
\text { within the bin } \\
1(<0.5 \mathrm{~mm})\end{array}$} & \multicolumn{2}{|c|}{$\begin{array}{c}\text { Percentage of MLC } \\
\text { position error } \\
\text { counts within the } \\
\text { bin } 2(\geq 0.5 \mathrm{~mm})\end{array}$} \\
\hline & $100 \%$ & $20 \%$ & $30 \%$ & $40 \%$ & $100 \%$ & $20 \% 30 \% 40 \%$ \\
\hline $2 \mathrm{~m}$ & 98. & & 96. & & 1.69 & 5.973 \\
\hline $4 \mathrm{~m}$ & 98.15 & 95.26 & 97.23 & 97.71 & 1.85 & \begin{tabular}{|l|l|}
4.74 & 2.7 \\
\end{tabular} \\
\hline $6 \mathrm{~mm}$ sweep & 98.62 & 96.21 & 97.32 & 97.94 & 1.38 & 3.792. \\
\hline $10 n$ & 98.26 & 97.38 & 97.41 & 97.82 & 1.74 & \begin{tabular}{ll|l}
2.62 & 2. \\
\end{tabular} \\
\hline $14 \mathrm{~mm}$ sweep & 98.76 & 97.14 & 97.58 & 97.70 & 1.24 & \begin{tabular}{|l|l|l|}
2.86 & 2.4 \\
\end{tabular} \\
\hline $16 \mathrm{~mm}$ sweep & 98.51 & 96.90 & 98.26 & 98.68 & 1.49 & $\begin{array}{lll}3.10 & 1.7 \\
\end{array}$ \\
\hline $20 \mathrm{~mm}$ sweep & 98.14 & 95.40 & 96.46 & 96.64 & 1.86 & 4.603 .5 \\
\hline Overall mean & 98.39 & 96.05 & 97.30 & 97.81 & 1.61 & \begin{tabular}{|l|l|l|l|} 
& 2.7 .95 & 2.19 \\
\end{tabular} \\
\hline
\end{tabular}

From the DynaLog analysis data for the sweep fields, the overall mean of percentage of the MLC positional error counts for the deviation less than $0.5 \mathrm{~mm}$ values are $98.39 \%$, $96.05 \%, 97.30 \%$, and $97.80 \%$ for non-gated, $20 \%$ DC, $30 \%$ DC, and $40 \%$ DC gated, respectively. These results show that the percentage of the MLC position error counts decreases as the gating duty cycle increases. The individual sweep field DynaLog file analysis data also clearly show the same trend of the increasing the duty cycle reducing the percentage of the MLC position error counts. These data clearly show that the increasing duty cycle increases the MLC positional accuracies and increases the delivery accuracy.

\section{h2. IMRT plan delivery DynaLog file analysis}

We acquired totally 720 DynaLog files (180 DynaLog files of non-gated delivery and 540 files of gated delivery) of the 10 IMRT plans consisting of 60 fields that were analyzed in this study. The DynaLog data were acquired three times for each IMRT field of different cycles of gated and non-gated delivery simultaneously during the measurement of the IMatriXX planar dose measurements, portal dose image prediction measurements, and point dose measurements. We analyzed the DynaLog files using the Varian DFV software and found the percentage of the MLC positional error counts, average error RMS, and maximum error RMS for all 720 fields (180 DynaLog files of non-gated delivery and 180 files of each gated delivery). The percentage of MLC error counts were collected for three bins $(<0.5 \mathrm{~mm}, 0.5$ to $<1.0 \mathrm{~mm}$ and $\geq 1.00 \mathrm{~mm}$ ). We found the mean and standard deviations (SD) for the respective bins for all the acquired data. The error histogram bar chart with the error bar values of SD were plotted from the collected data for the gated and non-gated deliveries, as shown in figure 7 . From the data, in bin 2 and bin $3(0.5 \mathrm{~mm}$ to $<1.0 \mathrm{~mm}$ and $1.0 \mathrm{~mm}$ and above), the percentage of the MLC position error counts decreases, as the duty cycle increases from $20 \%$ to $30 \%$ and $40 \%$ and the percentage of the increase is very minimal. The duty cycle increases the positional error counts is bin 1 (less than $0.5 \mathrm{~mm}$ ) is increasing that implicit that the MLC positional errors are decreasing. The MLC positional inaccuracy increased owing to the frequent interruption of the beam in the gated delivery. We have statistically analyzed the Error histogram results keeping the non-gated as a reference using the paired t test, we found that there is no statistical deviation for the three different duty cycles of gated delivery and the p-value calculated was $<0.001$. This statistical analysis ensures that the error histogram results of gated deliveries are not significantly differ from the non-gated delivery.

DFV analysis of dynalog files data for the error RMS values, we found the mean and standard deviation (SD) of the maximum leaf 
error and average leaf error for each MLC bank to all 720 fields, and the data are plotted in bar chart with error bars of SD valves are shown in figure 8. The RMS values of the dynalog file analysis does not shown any significant deviation between the different duty cycles of gated delivery over the non-gated delivery. Error histogram and error RMS data are analyzed using the SPSS software to find the statistical significance using paired t test. We obtained the $\mathrm{p}<0.001$ of the DynaLog file analysis for error histogram and error RMS. Statistical analysis results ensures that the different duty cycles of gated delivery over the non-gated delivery does not shown any significant deviation between them.

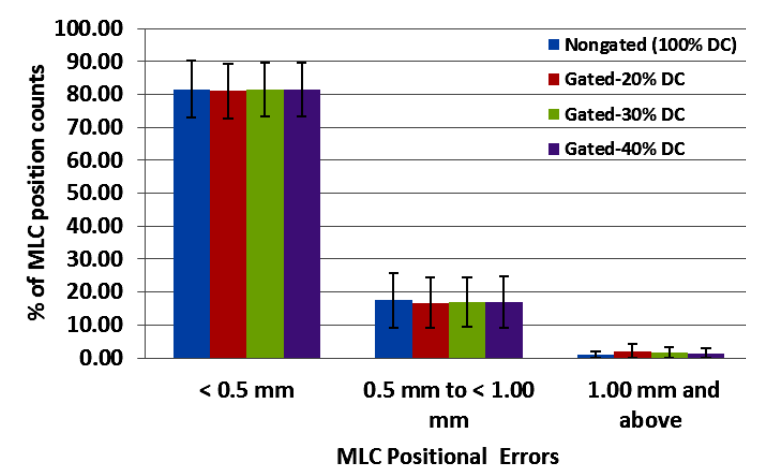

Figure 7. MLC DynaLog file analysis. Error histogram bar charts of the 60 IMRT fields for different duty cycles of gated and non-gated deliveries.

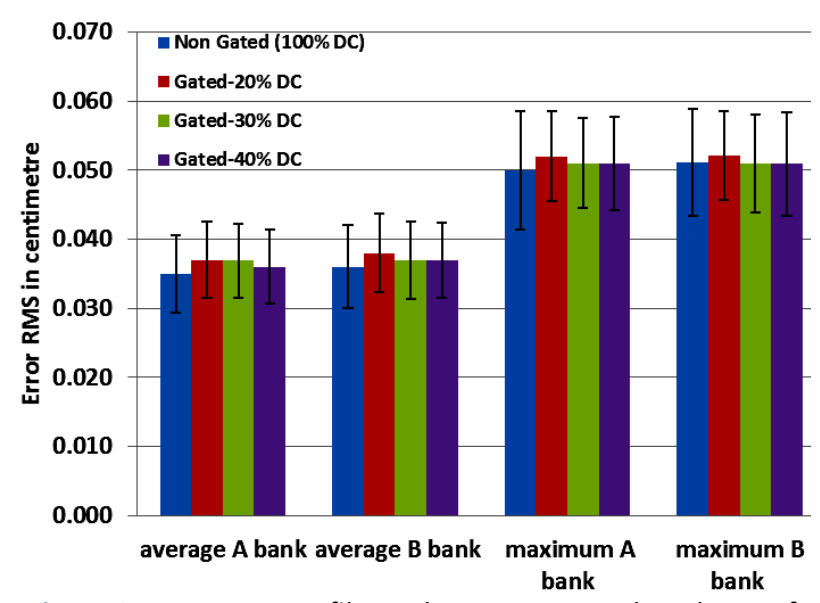

Figure 8. MLC DynaLog file analysis. Error RMS bar charts of 60 IMRT fields for different duty cycles of gated and non-gated deliveries.

\section{DISCUSSION}

Dosimetric characteristics for three different duty cycles (20\% DC, 30\% DC and 40\% DC) of gated delivery were compared with those of the non-gated delivery. MLC transmission values of gated deliveries were very well with the acceptable transmission value of $2 \%$ and the difference between the gated and non-gated deliveries are less than $0.3 \%$. Various authors are measured the DLG values of the Novalis Tx linear accelerator for the High Definition MLC (HD MLC 120) for 6 MV photon beams and our values of DLG for the gated and non-gated are closely agreeing with them $(25,27)$. In this study, we have not quantified the beam flatness and symmetry of the non-gated delivery. We have considered that the beam flatness and symmetry are very well within the limits of $2 \%$ for our Novalis Tx linear accelerator. We compared the different field size dose planes of the gated delivery with the non-gated delivery as a reference. This comparison enables us that the impact of frequent beam interruption (gated) changes the symmetry and flatness of the gated beam. Gamma pass percentage is more than 99\% between the gated and non-gated delivery. Gamma evaluation results implicit that the frequent beam interruption (gated delivery) does not alter the beam flatness and symmetry significantly. DMLC output ratio between the gated and non-gated delivery was measured for the different MLC sweeps. DMLC ratio measured in this study is less than the acceptable deviation $1.0 \%$. This DMLC output ratio suggests that the output of the linear accelerator for the gated deliveries is less than $0.62 \%$ deviation and this value is comparable with other studies $(19,20)$. From the obtained values in this work, we conclude that the MLC transmission, DLG, DMLC output, flatness, and symmetry values are comparable for the gated and non-gated deliveries.

The positional accuracy of MLC of the gated and non-gated deliveries was analyzed in this study for the static and dynamic MLC delivery methods. The EPID image of the picket fence test 
shows that the accuracy of static MLC positioning is less than $0.5 \mathrm{~mm}$ between the gated and non-gated delivery. The gamma evaluation analysis between the portal images of the picket fence test for the gated deliveries are agreeing $99.5 \%$ to the non- gated delivery in this study. Gamma analysis was carried out between the EPID images of the gated delivery to the reference on non-gated delivery. The gamma evaluation analysis between the portal images of the different sweep fields for the gated deliveries are agreeing $95.0 \%$ to the non- gated delivery in this study. The result shows that the deviation is more for the smaller sweep widths and the lesser duty cycle.

Gamma agreement improves the increasing sweep widths and the increasing duty cycle. This difference occurs due to the more number of MLC positions over the total beam-on time. For smaller the sweep widths the number of MLC control points is more and frequent abruption of beam and movements of MLCs cause this lesser gamma agreement when compare to the larger sweep widths and increasing the duty cycles of the gated delivery. However the gamma analysis for the sweep fields results are $<5 \%$ of gamma deviations and it is acceptable clinically. We observe the same kind of trend in the static and dynamic MLC delivery that the increase in duty cycle increases the gamma agreement and thus improves accuracy of delivery.

DynaLog files were collected for the different sweep fields of gated delivery to analyze the MLC positional error counts. We found that the MLC position errors are less than $1.0 \mathrm{~mm}$ for the dynamic sweep fields of different sweep widths. These lesser MLC positional deviation is attributed from the smoother and uniform MLC movements throughout the delivery. We observe that the similar kind of trend in the dynalog file analysis also, increase in duty cycle reduces the MLC positional errors and thus increases the plan delivery accuracy.

Non IMRT gated deliveries are analyzed for the Novalis Tx linear accelerator in this study and the gated deliveries were comparable with the non-gated deliveries. To analyze the gated IMRT delivery, we have taken 10 IMRT plans consists of 60 IMRT fields. Gamma analyses for the acquired dose planes were carried out under two different scenarios compared in this study: 1. Measured dose planes versus TPS calculated dose planes for the gated and non-gated deliveries to find the accuracy of the delivery over the planned IMRT plans/fields. 2. Non gated dose planes versus three different duty cycles of dose planes to find the impact of the gating technique on the gated IMRT delivery.

Table 5 presents the area gamma $\geq 1.0$ values of the mean and their associated standard deviations (SDs) for the three different duty cycles of gated deliveries of the 60 IMRT fields evaluated in our study for both the above mentioned scenarios. From the data, we observed that the $20 \%$ duty cycle showed more deviation than the other two duty cycles of gated delivery and non-gated delivery for all the fields analyzed in this study. We observed that the pass percentage of gamma increases with the increase in the duty cycle, which is a similar trend that what we observed for the DMLC sweep field gamma analysis. Both the EPID and IMatriXX analyses show similar trends for the gamma analysis; that is, the gamma pass percentage increases as the duty cycle increases. This deviation may be attributed to the frequent interruption of the beam and the lower MU per cycle for the $20 \%$ duty cycle compared to the $30 \%$ and $40 \%$ duty cycles. As the duty cycle increases, the frequency of beam interruption decreases and the MU per cycle is increases. Therefore, we obtain good agreement between the gated and non-gated deliveries for higher duty cycles. IMRT point dose measurements were also carried out for the gated and non-gated deliveries. The point dose measurements ratios are also confirmed that the increase in duty cycles increases the delivery accuracy. Several studies show the same trend that the increase in duty cycle increases the delivery accuracy of IMRT (6,9, 21-23). Dynalog file analysis of the gated IMRT clearly shows that the $20 \%$ duty cycle gated delivery is more MLC positional error counts than the $30 \%, 40 \%$ and non-gated deliveries. The highest number of MLC position error counts at the lower duty cycle delivery is due the frequent interruption of the MLC motions and its give rise the reduction 
in the accuracy of IMRT delivery. So while selecting the gating window for the treatment one should keep in mind that the decrease in gating duty cycle reduces the IMRT delivery accuracy. The statistical analysis between the gated deliveries over the non-gated delivery does not shown any statistical significant for the results of gamma evaluation analysis and the Dynalog file analysis. So the intended delivery of the IMRT for the gated deliveries is statistically significant.

\section{CONCLUSION}

MLC transmission, DLG, MLC positioning accuracy, and beam profile analysis results show no significant deviation between the gated and non-gated deliveries. The IMRT field gamma evaluation results suggest that the non-gated delivery and gated deliveries of different duty cycles are comparable with the TPS-calculated dose planes. These results show that the Novalis $\mathrm{Tx}$ linear accelerator gated operations maintained the intended IMRT dose delivery.

A gamma comparison of the gated delivery with the reference of non-gated delivery results exhibits that the increase in duty cycle reduces the deviation between the gated and non-gated deliveries. DynaLog file analysis data also shows that the increase in duty cycle reduces the deviation between the gated and non-gated deliveries. From the obtained results, we found that gating duty cycles might affect the accuracy of IMRT beam delivery to a certain extent, although these deviations are not significant as per this study up to a duty cycle of $20 \%$ for gated delivery. We plan to extend this study in the future for gated delivery duty cycles less than $20 \%$ to analyze whether they cause any significant changes in the IMRT delivery accuracy.

\section{Conflicts of interest: Declared none.}

\section{REFERENCES}

1. Jiang SB (2006) Technical aspects of image-guided respira682 tion-gated radiation therapy. Med Dosim, 31: 141-151.

2. Bortfeld T (2006) IMRT: a review and preview. Phys Med Biol, 51: 363-379.

3. Keall P, Vedam S, George R, Bartee C, Siebers BSJ, Lerma F, Weiss E, Chung T (2006) The clinical implementation of respiratory-gated intensity modulated radiotherapy. Med Dosim, 31: 152-162.

4. Chavaudra J and Bridier A (2001) Definition of volumes in external radiotherapy: ICRU reports 50 and 62. Cancer Radoatherapie, 5: 472-478.

5. Purdy JA (2004) Current ICRU definitions of volumes: limitations and future directions. Semin Radiat Oncol, 14: 2740.

6. Chang Z, Liu TH, Cai J, Wang Z, Yin FF (2011) Evaluation of integrated respiratory gating systems on a Novalis Tx system. J Appl Clin Med Phys, 12: 71-79.

7. Vedam SS, Kaell PJ, Kini VR, Mohan R (2001) Determining parameters for respiration-gated radiotherapy. Med Phys, 28: 2139-2146.

8. Chang-Li R, Yu-xin C, Lu-Zhou W, WU-bing, Qi-bin S (2015) The influence of respiratory motion on dose distribution of 3D-CRT and IMRT- A simulation study. Int J Radiat Res, 13: 39-43.

9. Duan J, Shen S, Fiveash JB, Brezovich IA, Popple RA (2003) Dosimetric effect of respiration-gated beam on IMRT delivery. Med Phys, 30: 2241- 2252.

10. Swamy T, Radha A, Kathirvel, Subramanian (2016) Performance evaluation of gated volumetric modulated arc therapy. Int J Radiat Res, 14: 81-90.

11. Keall PJ, Mageras GS, Balter JM, Emery RS, Foster KM, Jiang SB, Kapatoes JM, Low DA, Murphy MJ, Murray BR, et al. (2006) The management of respiratory motion in radiation oncology report of AAPM Task Group 76. Med Phys 33: 3874-3900.

12. Giraud P and Houle A (2013) Respiratory Gating for radiotherapy: Main technical aspects and clinical benefits. Hindwai Publishing Corporation ISRN Pulmonology, 2013: 1-13.

13. Varian Medical Systems (2007) RPM Respiratory Gating system - Reference Guide.

14. Varian Medical Systems (2007) DMLC - Implementation Guide.

15. Varian Medical Systems (2008) DynaLog File Viewer Reference Guide.

16. Agnew CE, King RB, Hounsell AR, Mc Garry CK (2012) Implementation of phantom less IMRT delivery verification using Varian Dynalog files and R/V output. Phys Med Biol, 57: 6761-6777.

17. Calvo-Ortega JF, Teke T, Moragues S, Pozo M (2014) A Varian Dynalog file based procedure for patient dose volume histogram based IMRT QA. J Appl Clin Med Phys, 15: 100-109.

18. Litzenberg DW, Moran JM, Benedick AF (2002) Verification of dynamic and segmental IMRT delivery by dynamic log file analysis. J App Clin Med Phys 3: 63-72.

19. Ramsey CR, Cordrey IL, Oliver AL (1999) A comparison of beam characteristics for gated and non-gated clinical X-ray beams. Med Phys, 26: 2086-91.

20. Krimimski S, Li AN, Solberg TD (2006) Dosimetric characInt. J. Radiat. Res., Vol. 19 No. 3, July 2021 
teristics of a new linear accelerator under gated operation. J Appl Clin Med Phys, 7: 65-76.

21. Hugo GD, Agazaryan N, Solberg TD (2002) An evaluation of gating window size, delivery method, and composite field dosimetry of respiratory gated IMRT.

Med Phys, 29: 2517-2525.

22. Kubo HD and Wang $L(2000)$ Compatibility of Varian $2100 \mathrm{C}$ gated operations with enhanced dynamic wedge and IMRT dose delivery. Med Phys, 27: 1732-1738.

23. Ahunbay E and Li XA (2007) Investigation of the reliability, accuracy and efficiency of gated IMRT delivery with a commercial linear accelerator. Med Phys, 34: 2928-2938.

24. Berbeco RI, Neicu T, Rietzel E, Chen GTY, Jing SB (2005) A technique for respiratory-gated radiotherapy treatment verification with an EPID in cine mode. Phys Med Biol 50: 3669-3679.

25. Shende R and Patel G (2017) Validation of Dosimetric Leaf Gap (DLG) prior to its implementation in Treatment Planning System (TPS): TrueBeam ${ }^{\mathrm{TM}}$ millennium 120 leaf MLC.
Rep Prac Oncol Radiather 22: 485-494.

26. Balasingh STP, Rabi Raja Singh I, Mohamathu Rafic K, Ebenezer Suman Babu S, Ravindran BP (2014) Determination of dosimetric leaf gap using amorphous silicon electronic portal imaging device and its influence on intensity modulated radiotherapy dose delivery. J Med Phys, 40: 129.135.

27. Mullins J, De Blois F, Syme A (2016) Experimental characterization of the dosimetric leaf gap. Biomed Phys Eng Express, 2: 1-9.

28. Varian Medical Systems (2007) MLC Shaper - Instructions for Use.

29. Low DA and Dempsey JF (2003) Evaluation of the gamma dose distribution comparison method. Med Phys, 30: 2445 -2464 .

30. Winiecki J, Morgas T, Majewska K (2009) The gamma evaluation method as a routine QA procedure of IMRT. Rep Pract Oncol Radiother, 14: 162-168. 
